\title{
SpS1-Spectroscopic observations of young disk evolution with Herschel and ALMA
}

\author{
W. R. F. Dent \\ ALMA JAO, Santiago, Chile \\ email: wdent@alma.cl
}

\section{Introduction}

In the next few years, both Herschel and ALMA will be providing unique new insights into the physics and chemistry of protoplanetary disks. In particular, they will be used to study how disks evolve from massive embedded systems around young Class 0 objects, through low-mass disks around optically-visible T Tauri stars, to debris disks around stars on the main-sequence. Gas dominates the mass in the younger systems, but in debris systems there is very little - if any. How does the gas disappear, what is the effect of this on planetary formation, and what is the role of "transition" disks? I outline some of the areas where these two large facilities will contribute to these studies, focussing on the Herschel Key project, GASPS, and looking forward to the role of ALMA.

\section{Herschel and GASPS}

Herschel is a $3.5 \mathrm{~m}$ telescope launched in early 2009 , and currently at the L2 point, $1.5 \times 10^{6} \mathrm{~km}$ from Earth. It has three cryogenic instruments operating in the far-infrared. Focussing on the spectroscopic capabilities of these instruments, HIFI is a heterodyne receiver for 480-1250 and 1410-1910 GHz (de Graauw et al., 2008), PACS is an instrument with a 5x5 IFU of resolution $\sim 2000$, operating in the region 60-200 $\mu \mathrm{m}$ (Poglitsch et al., 2008), and SPIRE includes an imaging FTS of resolution up to 1000 for the 200-700 $\mu \mathrm{m}$ region (Griffin et al., 2008). Several large programs studying disks with spectroscopy have been allocated time, and it is likely that many more open-time projects will be using these instruments during the spacecraft lifetime. The current large projects include a study of the nearby "Fab-4" debris disks, HOBYS (a study of massive young stars), WISH (studying water in young star-forming regions), a HIFI spectral survey of young stellar objects, GASPS (studying gas in protoplanetary systems), DIGIT (dust, ice and gas in young systems) and HOPS (observing protostars in Orion). We focus here on GASPS, which is tailored to study the evolution of gas and dust in protoplanetary disks.

The aim of GASPS is to study several key gas tracers and dust in a wide range of visible young stellar objects. The sample of $\sim 200$ targets covers a range of stellar types, disk dust mass, age, UV \& X-ray flux, and $\mathrm{H} \alpha$ strength. The species to be observed are [OI] $(63$ and $145 \mu \mathrm{m})$, [CII] $(157 \mu \mathrm{m})$ and lines of $\mathrm{H}_{2} \mathrm{O}$ at 78 and $180 \mu \mathrm{m}$; these are predicted to be the brightest in such objects. The targets are selected from the nearest star formation regions and associations of ages from 1 to $30 \mathrm{Myr}$, including Taurus, $\beta$ Pic Moving Group, $\eta$ Cha, Tuc Hor and Upper Sco. They are chosen to have relatively low extinction, in order to minimise contamination from nearby cloud emission. Line strengths and ratios will be used to derive physical parameters, by comparison with an extensive model database (Woitke et al., 2009). PACS will not have sufficient spectral resolution to resolve the lines; therefore, the survey will rely on comparison with these models, with dust SEDs, and with other gas species. From this, we will investigate trends over the sample, looking at what parameters affect the line strengths and ratios. GASPS is a survey, and will provide line and continuum strengths. But to derive detailed information about individual disks, both spectral and spatial resolution are necessary; this is what ALMA will provide.

\section{ALMA and future studies of gas in disks}

ALMA will be a 66-element interferometer, operating at wavelengths from $350 \mu \mathrm{m}$ to $9 \mathrm{~mm}$ at an altitude of $5 \mathrm{~km}$ in northern Chile. It will have movable antennas and baselines up to $10 \mathrm{~km}$, providing resolutions from 1 arcsec down to less than 20 mas at what is probably the prime 


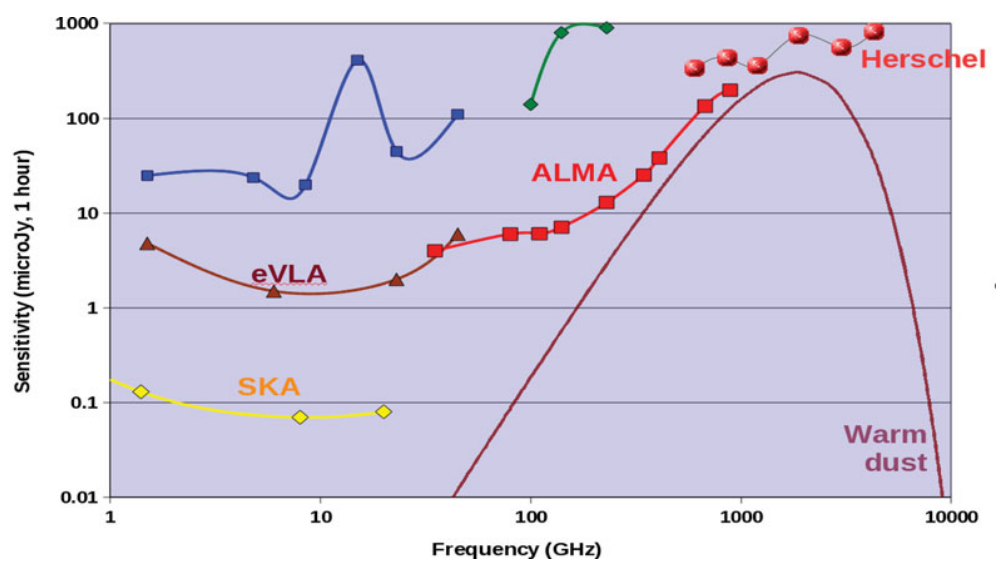

Figure 1. Sensitivity of ALMA, Herschel and other facilities. The upper lines (= least sensitive) refer to existing instruments, the solid curve shows a typical SED from warm dust.

observing band, $850 \mu \mathrm{m}$. The gain over existing interferometers will be in three main factors: resolution $(\sim 20 \mathrm{x})$, sensitivity $(20 \mathrm{x}$ more baselines) and sensitivity $(\sim 40 \mathrm{x})$. Figure 1 compares the sensitivity of ALMA with other facilities.

ALMA will be ideal for studying the structure, chemistry and physics of protoplanetary and debris disks, which requires improvements in all three factors to make significant advances. In particular, chemical differentiation, turbulence, in/outflows will be seen on scale of a few AU in the nearest star-formation regions. As well, it should reveal non-axisymmetric disk structure, in both self-gravitating disks and in systems affected by young protoplanets (e.g. Narayanan et al., 2006). The study of dust-rich debris disks should be significantly advanced by ALMA: detection of 1 Kuiper Belt's mass of dust, of the remnant CO and CI in young debris disks (Kamp et al., 2003), and the detection of classical debris disks in Taurus will be possible. The resolution should allow study of clumps on sub-AU scales in disks, looking at structure and motions in these systems.

ALMA will be fully operational by 2013. However, an opportunity to perform early science will be available in 2011. At that time the number of antennas will be no less than 16, and baselines will be limited to $500-1000 \mathrm{~m}$. The resolution will be $\sim 0.5 \operatorname{arcsec}$ at $850 \mu \mathrm{m}$ with sensitivity already significantly improved on existing facilities.

\section{References}

de Graauw et al., 2008, SPIE 7010, 4

Griffin et al., 2008, SPIE 7010, 6

Kamp et al., 2003, A\&A 397, 1129

Narayanan et al., 2006, ApJ 647, 1426

Poglitsch et al., 2008, SPIE 7010, 5

Woitke, Kamp, \& Thi 2009, A\&A 501, 383 